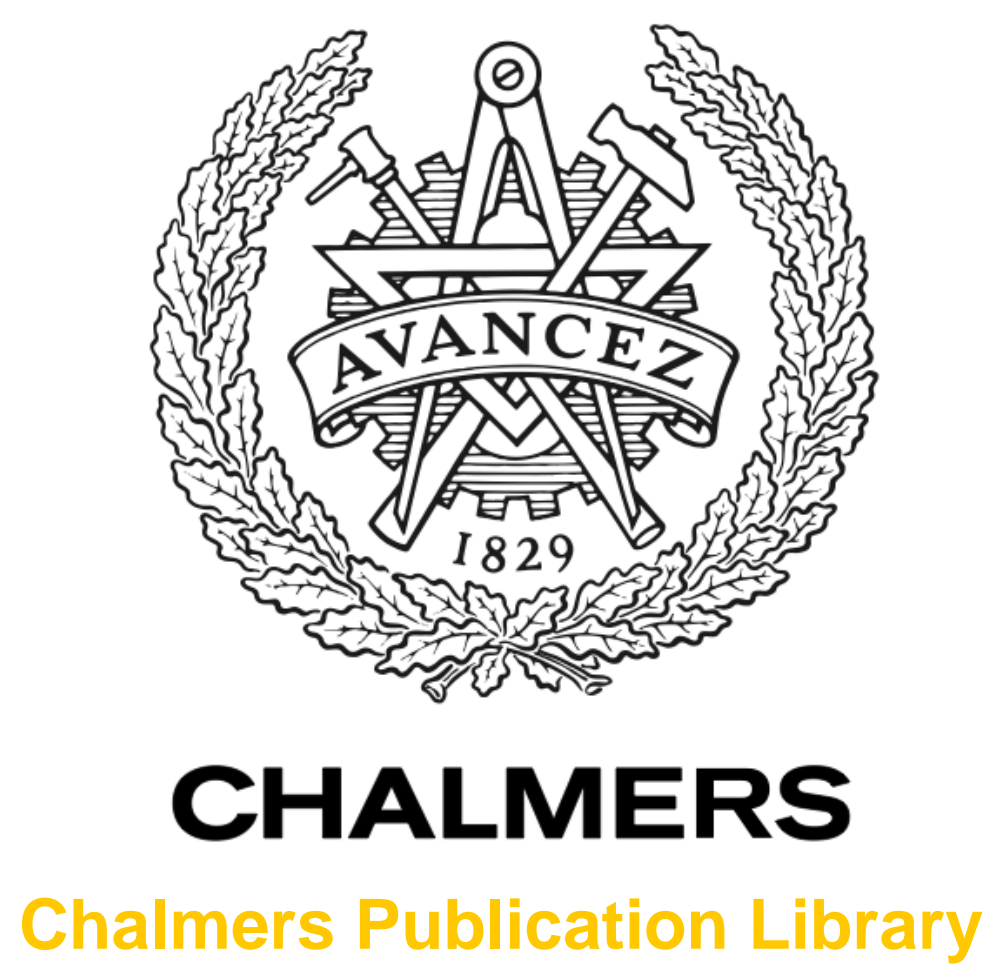

Four-Dimensional Modulation Formats for Long-Haul Transmission

This document has been downloaded from Chalmers Publication Library (CPL). It is the author's version of a work that was accepted for publication in:

Optical Fiber Communication Conference (OFC) 2014

Citation for the published paper:

Johannisson, P. ; Sjödin, M. ; Eriksson, T. (2014) "Four-Dimensional Modulation Formats for Long-Haul Transmission". Optical Fiber Communication Conference (OFC) 2014 pp. M2C.4.

Downloaded from: http://publications.lib.chalmers.se/publication/195299

Notice: Changes introduced as a result of publishing processes such as copy-editing and formatting may not be reflected in this document. For a definitive version of this work, please refer to the published source. Please note that access to the published version might require a subscription. 


\title{
Four-Dimensional Modulation Formats for Long-Haul Transmission
}

\author{
Pontus Johannisson, Martin Sjödin, Tobias A. Eriksson, and Magnus Karlsson \\ Photonics Laboratory, Chalmers University of Technology, SE-412 96 Göteborg, Sweden \\ pontus.johannisson@chalmers.se
}

\begin{abstract}
A number of four-dimensional modulation formats are described theoretically and from an implementation point of view. The requirements for digital signal processing and the trade-off between spectral efficiency and receiver sensitivity is discussed.

OCIS codes: (060.1660) Coherent communications; (060.4080) Modulation; (060.2330) Fiber optics communications
\end{abstract}

\section{Introduction}

A coherent receiver performs a linear mapping of the four-dimensional (4D) optical signal space to the electrical domain and this brings a large freedom in the choice of modulation format. This can be used, e.g., to make flexible systems with a fine granularity in the trade-off between data throughput and transmission reach. This has made 4D modulation an important topic lately and in this paper, we give an overview of a number of 4D modulation formats for long-haul transmission. In particular, we compare the digital signal processing (DSP) that is needed for different formats and discuss a number of experimental realizations.

\section{Modulation Formats}

In 2009, Bülow described POL-QAM [1] and Agrell and Karlsson reported the most power efficient 4D modulation format, the polarization-switched (PS-) QPSK [2]. The fundamental parameters of these formats are the spectral efficiency (SE) and the asymptotic power efficiency (PE) [2]. However, as the PE in general depends on the target bit error rate (BER) and the forward error correction (FEC), the choices of modulation format and FEC are connected.

Many important $4 \mathrm{D}$ modulation formats can be defined using $4 \mathrm{D}$ lattices. In the $Z_{4}$ lattice, each point has integer coordinates, and as subsets of $Z_{4}$ we find, e.g., polarization-multiplexed (PM) quaternary phase-shift keying (QPSK), PM rectangular 16-ary quadrature amplitude modulation (16-QAM), and other QAM constellations. PM-QPSK is popular for many reasons, including the transmitter and receiver simplicity, but the step between different QAM constellation is large, both in terms of SE and PE. For example, going from PM-QPSK to PM-16-QAM doubles the data rate but requires a significantly increased signal-to-noise ratio (SNR). The QAM modulation formats are represented by the blue line in Fig. 1, which shows the SE per polarization as a function of the sensitivity penalty $(1 / \gamma)$, i.e., the asymptotic PE loss compared to PM-QPSK [2].

For increased flexibility it is desirable to find formats with intermediate SE and PE values. The $D_{4}$ lattice represents the densest possible $4 \mathrm{D}$ packing and contains points with integer coordinates, where the sum of the coordinates is even. The $D_{4}$ lattice is related to $Z_{4}$ by the operation of set partitioning [3], where the number of constellation points is reduced by $50 \%$, i.e., one bit is lost, and the minimum distance between constellation points is increased by $\sqrt{2}$, leading to a higher PE. In this way, e.g., the $D_{4}$ formats PS-QPSK and 128-SP-QAM can be constructed from PMQPSK and PM-16-QAM, respectively. Compared to the generating constellations, the asymptotic PE is increased by $1.76 \mathrm{~dB}$ for PS-QPSK and $2.43 \mathrm{~dB}$ for 128-SP-QAM. The SP formats are represented by the green line in Fig. 1, and it is seen that they complement the SE/PE-values of the QAM formats very well.

Pulse-position modulation (PPM) increases the dimensionality, potentially far beyond four, and can be used to improve the sensitivity at the cost of a reduced SE [4]. For example, using $K$-PPM-PM-QPSK, a symbol consists of a PM-QPSK symbol transmitted in one selected slot out of $K$ consecutive ones. However, it should be noticed that applying PPM to a 4D symbol is not the same as applying polarization multiplexing to a $2 \mathrm{D}$ format that uses PPM. In Section 4 we discuss PM-2-PPM-QPSK, in which the positions of two QPSK symbols are individually selected. PM-2-PPM-QPSK is equivalent to PS-QPSK in terms of SE and PE.

A cross-polytope exists in any number of dimensions and contains all permutations of $\{ \pm 1,0,0, \ldots, 0\}$. This corresponds to the most power-efficient modulation format in one (binary phase-shift keying), two (QPSK), and four dimensions (PS-QPSK). It is conjectured that this is the most power-efficient format regardless of the dimensionality. One way to increase the dimensionality beyond four is to use frequency and polarization switched QPSK and this modulation format is discussed in Section 4. 


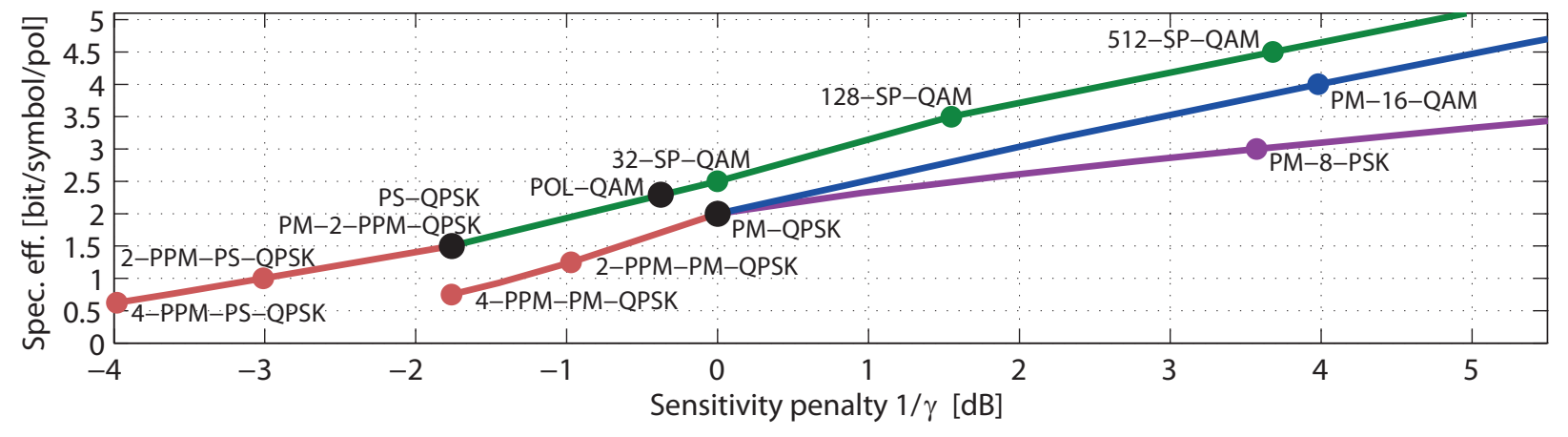

Fig. 1: The SE as a function of the PE penalty at low BER (compared to PM-QPSK) [2]. Included are POL-QAM, rectangular QAM (blue), SP formats (green), PSK (purple), and PPM (orange) using the formats at the ends of the lines. In practice, many more parameters are important: Modulation formats have differently complicated implementations and different tolerances to non-idealities, FEC influences both the performance and the DSP complexity etc.

\section{Digital Signal Processing}

The DSP that is needed in the receiver differs between modulation formats. If the aim is to make an elastic receiver with many possible choices of modulation formats, the use of training data can generalize the DSP greatly [5]. Here, we briefly discuss how the traditional DSP choice for PM-QPSK is affected when using other modulation formats.

Differential data encoding is often used to address the problem of cycle slips. This is straightforward for PM-QPSK and PM-16-QAM and viewing 128-SP-QAM as a subset of PM-16-QAM, a pragmatic choice is to use the same scheme as for PM-16-QAM [6]. The case for PS-QPSK is more difficult [7]. One way is described in [8], i.e., one bit is encoded in the selection of the polarization and two bits are differentially encoded in the phase of consecutive 4D symbols. A similar approach can be used for PM-2-PPM-QPSK, i.e., one bit is encoded in the selection of the time slot and two bits are encoded differentially in the phase.

Polarization demultiplexing/dynamic equalization is for PM-QPSK typically carried out using the constant modulus algorithm (CMA) [9], which also works for PM-16-QAM with reduced performance. Therefore, CMA is then often only used initially to enable a switch to decision-directed mode and we have used this also for 128-SP-QAM. However, the CMA does not work for PS-QPSK but the PS-CMA [10] or the scheme described in [11] can be used. For PM2-PPM-QPSK, we used a variation of the algorithm described in [12]. Thus, for each symbol and polarization a decision is made if a symbol is present. The so obtained algorithm is sensitive to the initial configuration [12]. It is not straightforward to extend CMA to work well for higher order PPM.

The Viterbi and Viterbi (V\&V) algorithm [13] is often used for phase estimation for PM-QPSK. The same algorithm can also be used for PM-16-QAM, but with significantly degraded performance. We have used the method based on test angles [14] for our PM-16-QAM and 128-SP-QAM experiments. For PM-2-PPM-QPSK, on average one out of two symbols carries power and only half of the symbols contribute to the phase estimation, but the V\&V algorithm still works. For PS-QPSK, there is the complication that the phases in the $x$ - and $y$-polarizations have to be locked for differential decoding. When a cycle slip occurs in one polarization, all following phase transitions otherwise become incorrect [8]. Thus, it is necessary to perform a joint phase estimation of the polarization-multiplexed signals. The relative phase between the polarizations must then be identified and this was done using the V\&V algorithm on a block of data from each tributary, see also [11].

Hard decision detection for all $Z_{4}$ and $D_{4}$ formats can be performed using the algorithm by Conway and Sloane [15]. This works both with absolute and differential encoding, and in the latter case differential decoding is performed after the decision. For PS-QPSK, one bit can be recovered by deciding which was the selected launch polarization [16] and the remaining two bits are then given by the differential phase between consecutive symbols. The situation for PM-2-PPM-QPSK is similar, with the roles of the two polarization states replaced by neighboring time slots.

\section{Experimental Implementation}

Many 4D modulation formats have been investigated experimentally. The first implementation of PS-QPSK was reported in 2011 [16]. The transmitter consisted of an IQ-modulator and two amplitude modulators in a push-pull con- 
figuration to generate QPSK symbols and to switch the polarization, respectively. At the same bit rate, the $1.0 \mathrm{~dB}$ sensitivity gain of PS-QPSK over PM-QPSK at a BER $=10^{-3}$ was confirmed and it was shown that the two modulation formats have similar nonlinear tolerance. In a WDM experiment with nine $50 \mathrm{GHz}$-spaced channels, PS-QPSK and PM-QPSK were compared in terms of the transmission reach, using three different fiber span lengths [8]. Compared to PM-QPSK, the use of PS-QPSK increased the reach with more than $41 \%$ for the same symbol rate (20 Gbaud) and $21 \%$ for the same bit rate. Comparing $80 \mathrm{WDM}$ channels at a symbol rate of $28 \mathrm{Gbaud}$, a $Q^{2}$-factor improvement close to $3 \mathrm{~dB}$ was found in both dispersion-managed and uncompensated optical links [11]. The reduced data throughput can be motivated if the received signal quality is too low to allow PM-QPSK transmission.

POL-QAM, which is also known as 6PolSK-QPSK, has been implemented at 28 Gbaud in a WDM experiment using a transmitter based on a digital-to-analog converter (DAC) [17]. Comparing with PM-QPSK, it was seen that the implementation penalty was higher $(3.4 \mathrm{~dB}$ and $2.1 \mathrm{~dB}$, respectively) and that the transmission reach was reduced by $22 \%$. However, the POL-QAM channel transmitted $12.5 \%$ more data, again showing that optimum performance can only be obtained by considering both modulation formats, FEC, and DSP/hardware limitations.

Two simultaneously published transmission experiments have compared 32-SP-QAM with a hybrid of PM-QPSK and PM-8-QAM, using $50 \%$ of each kind of symbols $[18,19]$. Both these modulation formats carry 5 bits per 4D symbol, making a direct comparison straightforward. It was found that 32-SP-QAM performed slightly better (except when using unrealistically high laser linewidth). Together, these publications show that 32-SP-QAM is a realistic alternative but again highlights the need for a DSP generalization.

128-SP-QAM has been compared with PM-16-QAM at a symbol rate of 10.5 Gbaud [20]. It was found that the transmission reach can be increased by $50 \%$ at the cost of a bit rate reduction of $1 / 8$. This agrees well with the numerical results found in [6,21]. Simultaneously with [20], an interesting study of a WDM system was published [22]. In this work, both 128-SP-QAM and 32-SP-QAM were implemented at 28 Gbaud using a DAC-based transmitter. This allowed rectangular shaping of the channel spectra with small penalty in a back-to-back measurement $(1.5 \mathrm{~dB}$ and $0.8 \mathrm{~dB}$ penalty, respectively, at $\mathrm{BER}=4 \times 10^{-3}$ ). Measured at the same symbol rate, stepping from PM-16-QAM to 128 -SP-QAM allowed $2.7 \mathrm{~dB}$ increase in reach, and continuing to 32-SP-QAM increased the reach $3 \mathrm{~dB}$ further.

PPM can achieve very high sensitivity as demonstrated by Liu et al. using 16-PPM-PM-QPSK [23]. However, PPM does not necessarily need to be combined with PM-QPSK [4] and one interesting format is PM-2-PPM-QPSK, which is equivalent to PS-QPSK in terms of SE and PE. In one experimental implementation, it was shown that compared at the same bit rate and at a BER $=3.8 \times 10^{-3}$, the reach can be increased by $40 \%$ over PM-QPSK [24].

One modulation format that corresponds to the cross-polytope in eight dimensions is the frequency and polarization switched QPSK, 4FPS-QPSK [25]. Using two subcarriers, the frequency, polarization, quadrature, and polarity are individually switched. (The two last choices correspond to the QPSK symbol.) In this way, the symbol alphabet consists of all permutations of $\left\{ \pm 1,0^{7}\right\}$. 4FPS-QPSK has $3 \mathrm{~dB}$ higher asymptotic PE than PM-QPSK and half the spectral efficiency. The experiment showed an $84 \%$ increase in transmission reach over PM-QPSK.

\section{Conclusion}

In conclusion, we have discussed a number of 4D modulation formats for long-haul transmission. Experimental implementations show that the choice of modulation format, the performance and computational complexity of the FEC, and the hardware limitations are essential to consider jointly for optimal performance. The differences in DSP required for different formats suggests that using training data may be the simplest way to make a flexible system.

\section{References}

[1] H. Bülow, OFC 2009, p. OWG2.

[2] E. Agrell et al., J. Lightw. Technol. 27, 5115 (2009).

[3] G. Ungerboeck, IEEE Trans. Inform. Theory IT-28, 55 (1982).

[4] M. Karlsson et al., ECOC 2011, p. Tu.6.B.6.

[5] J. K. Fischer et al., ECOC 2013, p. Tu.3.C.1.

[6] M. Sjödin et al., Opt. Express 20, 8356 (2012).

[7] P. Johannisson et al., SPPCom 2013, p. SPM3D.4.

[8] M. Sjödin et al., Opt. Express 20, 7544 (2012).

[9] D. N. Godard, IEEE Trans. Commun. 28, 1867 (1980).

[10] P. Johannisson et al., Opt. Express 19, 7734 (2011).

[11] J. Renaudier et al., J. Lightw. Technol. 30, 1312 (2012).

[12] D. S. Millar et al., Opt. Express 19, 8533 (2011).
[13] A. J. Viterbi et al., IEEE Trans. Inform. Theory IT-29, 543 (1983).

[14] T. Pfau et al., J. Lightw. Technol. 27, 989 (2009).

[15] J. H. Conway et al., IEEE Trans. Inform. Theory IT-28, 227 (1982).

[16] M. Sjödin et al., Opt. Express 19, 7839 (2011).

[17] J. K. Fischer et al., ECOC 2012, p. We.1.C.4.

[18] R. Rios-Muller et al., ECOC 2013, p. Th.2.D.2.

[19] H. Sun et al., ECOC 2013, p. Th.2.D.3.

[20] T. A. Eriksson et al., OFC 2013, p. OTu3B.2.

[21] T. A. Eriksson et al., Opt. Express 21, 19269 (2013).

[22] J. Renaudier et al., OFC 2013, p. OTu3B.1.

[23] X. Liu et al., OFC 2011, p. PDPB1.

[24] M. Sjödin et al., OFC 2013, p. OTu2B.7.

[25] T. A. Eriksson et al., ECOC 2013, p. Th.2.D.4. 